\title{
LA TEMPORALIDAD, LA MEMORIA Y LA IDENTIDAD EN LA PROSA POÉTICA "LLUVIA" POR GASPAR OCTAVIO HERNÁNDEZ
}

\author{
The timeliness, memory and identity in the poetic prose \\ "Lluvia" by Gaspar Octavio Hernández.
}

Karina Ayodele Clarke Murrain*

\begin{abstract}
RESUMEN
El Istmo de Panamá es conocido empíricamente por su situación geográfica, su medioambiente y su clima, y no es sorprendente que el escritor y periodista afropanameño, Gaspar Octavio Hernández (1893-1918) usa el medioambiente y las condiciones temporales en Panamá para inspirar su escritura. Como escritor modernista, es plausible que Hernández fue atraído al proceso de producir poesía pero en su obra se nota que surgen también temas que se basan en los procesos de la naturaleza. Es decir, en el proceso de producir poesía el escritor emplea temas del proceso. Utilizando la crítica de Ángel Rama, en el artículo "Indagación de la ideología en la Poesía (Los dípticos seriados de Versos Sencillos)" y en su libro Rubén Darío y el modernismo, examinaré cómo Hernández en su prosa poética "Lluvia" explora y prueba el concepto de la identidad dentro del contexto geográfico único de Panamá.

Palabras clave: afropanameño, modernismo, poesía, naturaleza, memoria.
\end{abstract}

\begin{abstract}
Panama is known empirically for its geography, climate and natural environment so it is not surprising that the Afro-Panamanian writer and journalist, Gaspar Octavio Hernández (1893-1918) uses climate and natural conditions as inspirations for his writing. As a Modernist writer, it is entirely plausible that Hernández was attracted to and compelled by the process of writing poetry, rather that the product. As we approach his work critically, it is evident that Hernandez uses the poetic process to recreate processes in nature and the environment to inhabit his poetry. Simply, Hernandez's poetic process produces poetry with themes of process. Using the critical anchors of Angel Rama in his article "Indagación de la ideología en la Poesía (Los dípticos seriados de Versos Sencillos)" and in his book, Rubén Dario y el modernismo, this article will examine how Hernández, in his poetic prose of "Lluvia" explores and evinces the concept of identity within the unique geographical context of Panama.
\end{abstract}

Key Words: Afro-Panamanian, modernism, poetry, nature, memory.

Ph.D. (candidata), University of Nebraska-Lincoln. Estados Unidos.

Correo electrónico: karinaclarcke@me.com

Recepción: 04/03/2014. Aceptación: 30/06/2014. 
Debatiblemente, el modernismo en Panamá tiene como eje la obra de Gaspar Octavio Hernández (1893 - 1918). De muy humildes raíces el autodidacta Hernández se convirtió en poeta y luego periodista, y se ocupó de publicar sus versos y su prosa en las revistas corrientes de la época. Panamá tenía y mantenía una tradición del periodismo muy progresivo desde la primera independencia de España en $1821^{1}$. La información diseminada tuvo la responsabilidad de informar, educar y más importante apoderar a la gente. En Panamá llegaron la independencia de Colombia junto con el modernismo y el resultado fue que el periodismo en Panamá tuvo un tipo de renacimiento y surgen en el Istmo una multitud de periódicos y revistas. Como en 1821, la actividad el periodismo en Panamá apoyó el nacionalismo claramente panameño, frente a las influencias políticas, económicas y sociales, de Colombia, los Estados Unidos, las Antillas y Europa que amenazarían el sentido de la autonomía tanta anhelada por los panameños durante los años entre la primera y la segunda independencias.

La identidad y el nacionalismo todavía son asuntos que no están completamente resueltos en el Istmo de Panamá pero es evidente que la búsqueda de la identidad es central en cuanto al desarrollo y el establecimiento de la literatura en el país. La poesía en prosa titulada "Lluvia" por Gaspar Octavio Hernández es un ejemplo del acercarse a explorar la identidad istmeña. Aparece en la edición número 1 de la revista Esto y Aquello con la fecha del 15 de agosto 1914. Esta fecha es llamativa porque la primera publicación de la revista coincide con la apertura oficial del Canal de Panamá (Araúz 1996: 86²), el logro de ingeniería que empíricamente definiría la identidad de los panameños desde aquel momento en adelante. En la portada de la primera publicación, aparece el soneto "De mi tierra" por Enrique Geenzier, otro modernista panameño. Brevemente, el soneto celebra un día de julio en Panamá y la celebración de la fecundidad de la tierra; el poema menciona la flora y la fauna del Istmo y termina con la mención de una comida típica de Panamá, el tamal de gallina. El soneto que aparece en la portada sutilmente sugiere que la revista va a enfocar en la tierra de Panamá y en los que vienen del Istmo. El soneto es una clara señal temática para la revista. Luego en la primera página aparece la nota editorial " $\mathrm{A}$ la cumbre!" donde los editores, entre varias frases animadas, alientan al lector con su propósito¡:

¡Henos aquí, caballeros cruzados del Ideal, gentiles
hombres del Arte, prestos a escalar la cima!
Venimos confiados en nuestros favorecedores, a
clavar en la cúspide del capitolio del oriflama
blanco del Progreso!... ¡Nada paralizará nuestros
esfuerzos! (...) Queremos el progreso del país y para
tan alto fin, solicitamos el apoyo de los verdaderos
patriotas, de los literatos, de los obreros, de los
capitalistas, de los institutores, de los estudiantes,
de los empleados públicos de cuantos se interesan
por la Prosperidad de la Nación! Y esto dicho, ¡a la
cumbre! (Geenzier y Benuzzi 1914: 2)

En esta nota editorial el propósito de la revista y los motivos de los editores están declarados que son simplemente actividades que contribuyen al progreso del país, por medio de las letras. El próximo texto que aparece es un texto sobre Emilia Pardo Bazán, escritora gallega y naturalista, "Conceptos sobre un libro" (Benuzzi 1914: 4-5) por Santiago Benuzzi. En este artículo Benuzzi celebra la obra naturalista de la escritora y reconoce su estilismo y su aportación a las letras naturalistas. A cambio de la expectativa de los modernistas, Benuzzi no critica ni rechaza el libro de Pardo Bazán, sino la reconoce con palabras fraternales y celebra su estética y su adiestramiento en el campo de naturalismo. El reconocimiento de una obra naturalista en un revista panameña es llamativo y creo que la naturaleza en cuanto al modernismo panameño es un tema principal. En las primeras páginas de la revista hay una celebración de la tierra de Panamá, luego un llamamiento para animar a los intelectuales de Panamá, y una celebración de una obra naturalista. Las páginas iniciales de la primera edición de Esto y Aquello ofrecen un cuadro sinóptico de la situación literaria, histórica y social en Panamá. Como la obra de Hernández, "Lluvia" también aparece en esta edición, significa por lo menos que él participaba en la comunidad literaria de Panamá y también que fue contribuidor integral 
en cuanto a las letras modernistas en Panamá de los escritores de la Primera Generación de la República ${ }^{3}$.

Gaspar Octavio Hernández nació Octavio Augusto Hernández el día 4 del julio del 1893. De acuerdo con Concha Peña en su biografía Poeta del pueblo, él fue el primer hijo de su padre, Federico Hernández, y el tercer hijo de su madre, Manuela Solanilla. Tuvo dos hermanos mayores, Esmeralda y Pablo, y dos menores, Adolfo y Dimas Eugenio. En 1914, el joven Hernández ya había experimentado una vida llena de abandono y tristeza. Cuando tenía once años, su padre ya había abandonado la familia, su madre había fallecido y uno de sus hermanos, Dimas Eugenio, se fue a vivir con una tía. En el momento de "Lluvia" se supone que Hernández, que fue producto de una familia fatalmente quebrada, creció con un sentido fugaz de la identidad; se supone, igual como el Istmo en aquel entonces, que Hernández sufría una crisis de identidad. Entonces, cuando aparece "Lluvia" en las primeras páginas de Esto y Aquello, parece una repuesta a la llamada de la nota editorial "¡A la cumbre!”

Panamá es conocido empíricamente por el medioambiente y el clima. En la prosa poética de "Lluvia," es evidente que Hernández emplea el acontecimiento de la lluvia y la experiencia con ella para explorar y probar una ideología de la identidad dentro del contexto geográfico único de Panamá. En aquel entonces la situación geográfica de Panamá y luego la facilidad que encontraron los poderes de los Estados Unidos, Europa y Colombia en explotarla (primero con el ferrocarril y luego con el Canal), la obra de Hernández funciona como desafío frente al interés extranjero. Hernández reconoce como puntos principales el hallar un sentido de la identidad y el priorizar un concepto de nacionalismo panameño. Es más plausible esa aseveración, al reconocer que la publicación de esta obra en la primera edición de Esto y Aquello del 15 de agosto del 1914 coincide con el día que abre el Canal de Panamá. Así, la obra de Hernández podría funcionar como antídoto a la maleza; la maleza siendo la lucha de mantener una consciencia de auto-actualización en la comunidad istmeña. Es aún más apreciada la obra de Hernández al considerar que coincide con el ambiente histórico cuando el Istmo estaba tratando de recuperar, o más bien establecer una identidad nacional. Asimismo, se supone que Gaspar Octavio Hernández, siendo afropanameño, huérfano, pobre y joven también estaba experimentando una crisis de identidad. En Hernández como modernista se nota que su obra refleja una separación y quizás un rechazo de las normas del Romanticismo europeo, por su originalidad; pero lo que hace su obra única y distintivamente panameña, es que Hernández consistentemente reconoce la naturaleza y centraliza su discurso basándose en la experiencia con la naturaleza. La obra de Hernández refleja que tiene un sentido de la identidad que está desarrollándose por medio de reconocer y celebrar la naturaleza y los procesos naturales. En este texto específico la lluvia tiene un papel desdoblado: por un lado es solamente una ocurrencia temporal y por otro lado, es un acontecimiento potente y catalizador en cuanto a los que presencian la lluvia. Como en Panamá la lluvia es una ocurrencia casi diaria, Hernández hábilmente transforma la ocurrencia prosaica de llover para ayudar a reconocer la experiencia climática como manera de desarrollar una ideología de la identidad.

El texto "Lluvia" relatada en la primera persona, describe un momento en un lugar supuestamente urbano e idealizado en Panamá. La descripción emula una obra naturalista pero la narración se revela como una reflexión personal e íntima y lector está invitado a experimentar el momento personal del narrador por medio de la palabra escrita y narrada. El título sencillo "Lluvia" está situado enigmáticamente contra la complejidad que el texto está por revelar. El título sucinto no ayuda al lector concebir el posible camino que va el discurso pero, a la vez, el lector puede considerar la arbitrariedad tanto dañina como beneficiosa que la caída de la lluvia puede abarcar. El texto empieza con la mención de la lluvia que cae con el ritmo musical, pero la voz narrativa atribuye calidad negativa al movimiento de la lluvia: "Con música dolorosa y monótona, que semeja una interminable 
lamentación, cae la lluvia." (Hernández 1914: 6) El texto relata la experiencia personal de la lluvia en el momento de llover y desde el principio se nota que toda la acción del texto se basa en la lluvia, reconociéndola como el protagonista de la narración:

\begin{abstract}
A través de una de las grandes ventanas de mi aposento de enfermo, yo la miro descender y, pienso que para mí nada hay tan inexplicablemente consolador como escuchar desde mi tibio lecho ese cántico intenso- lento y desconsolador como la última oración de un reo- que la lluvia murmura, ya en el techo de zinc de mi casa; ya en el patio concreto da la mansión contigua a la mía; ora en la tierra negra, sembrada de rosales, del bello jardín que frente a mi habitación se extiende con la seductora policromía de sus flores; ya en los tersos ladrillos que cubren de inmensa capa rojiza la sinuosa extensión de la calle. (Hernández 1914: 6)
\end{abstract}

La primera frase establece el ambiente lírico para el texto y luego todo el discurso en el primer párrafo gira alrededor de los efectos reales de la lluvia. La poética en la primera frase revela un tipo de rima asonante interior y da una sensación de un suspiro cargado de emoción. La frase en sí funciona como una lamentación y la asonancia de la vocal $a$ poéticamente suplica la expresión física de una boca abierta. La primera frase también indica que es un momento corriente y en tal presente poético, el uso del presente indicativo tanto refuerza la sensación del presente como implica una rutina. La rutina, más específicamente, se referiría al proceso natural y cíclico de la ocurrencia de lluvia inclusive la repetición de las gotas de lluvia al caer. La lluvia, como el título implica y como la primera frase refuerza, surge como protagonista (sujeto) en el texto. La lluvia está reconocida como un proceso potente con la capacidad de producir música y existe en el mismo nivel como el compositor, el músico o los instrumentos. La música, que es una referencia cultural, ahora existe en este texto como un sonido de índole completamente natural. Además, la narración parece reconocer que la lluvia como proceso no es como un dolor, sino la música en el papel de protagonista, produce el dolor. La comparación entre la caída de la lluvia y la lamentación refuerza dos niveles del presente poético: el primero es el nivel de lo corriente y lo ambiental; y el segundo es el nivel de lo nostálgico y lo emocional.

La segunda frase tiene dos funciones, porque introduce la voz narrativa y forma totalmente el resto del primer párrafo. En esta frase, en forma de párrafo, la lluvia está en la posición de un proceso observado, admirado y empoderado. No es solamente la caída de la lluvia sino la lluvia parece como compañera y el narrador que observa la lluvia se encarga de transmitir su experiencia por medio de las palabras. El proceso de la lluvia adquiere calidad eterna porque la descripción en el presente indicativo señala la rutina, lo repetitivo y lo cíclico. La experiencia descrita se revela de manera cinemática y el lector junto con el narrador hallan un suceso de dos puntos medioambientales: la lluvia afuera y la vista de ella desde la ventana de un cuarto interior de una casa. Los dos espacios, una afuera (de la lluvia) y otro adentro (del narrador) están vinculados por medio de la observación desde el interior, pero reconoce que la observación fue catalizada por un proceso afuera. En este juego visual la lluvia es el catalizador que causa que el narrador observe/contemple, luego surgen entendimiento/interpretación y el resultado, o sea el producto del proceso es el texto. El poder de la lluvia no se basa solamente en la lluvia y su capacidad de producir música sino la lluvia también es consoladora, es espiritual y es, sobre todo, omnipresente desde el punto de vista del narrador. De manera meta-literaria, la largura de la segunda frase parece emular exactamente lo que el narrador observa de la lluvia y cómo interacciona con el medioambiente. Aunque es un momento corriente hay una sensación "sin tiempo" porque el movimiento y el toco de la lluvia indican que no está detenida y parece que las palabras en el párrafo compiten para representar adecuadamente la verdadera experiencia que observa el narrador. Además, la frase revela la aparente universalidad de la experiencia del narrador porque concentra la narración no tanto en el medioambiente sino enfoca en la caída de la lluvia y lo que cataliza. En el primer plano, 
la lluvia se asocia con acciones sencillas, pero aunque la narración está en primera persona, el discurso enfoca en la acción de la caída de la lluvia y es evidente que para la voz narrativa la lluvia tiene impacto diverso. El primer párrafo refleja el impresionismo del narrador: la lluvia es musical, dolorosa y lamentosa; la lluvia es también potentemente consoladora y es un poco hechicera; y finalmente la lluvia es omnipresente porque el toco es universal (las ventanas, el techo, el patio, el jardín, etc.) Hay que considerar que cuando cae la lluvia no discrimina sino es igualitaria; asimismo, la lluvia es una experiencia común y por eso es universal. Al final del primer párrafo se nota que el narrador en vez de ser sujeto, se transforma en objeto que está incluido en el conjunto de los objetos mencionados en la lectura.

El segundo párrafo del texto revela que el narrador se ha dado cuenta de algunas impresiones por su experiencia con la lluvia:

\footnotetext{
Vosotros, los que no sois solteros ni viudos ¿no veis cómo en las noches de lluvia, cuando el cierzo canta un tiernísimo himno de penas en las puertas de vuestras casas, os miran con más pasión los ojos de la mujer idolatrada, como si ella presumiendo que estáis friolentos, quisiera daros calor con ellos? (Hernández 1914: 6)
}

La idea de la universalidad está reforzada al considerar esta cita del segundo párrafo. En el primer párrafo la lluvia aparece como protagonista que engendra una experiencia en el narrador y hasta tal momento en el texto sólo hay una interacción entre dos elementos, la lluvia y el narrador. Pero en el segundo párrafo otro elemento está introducido, el vosotros, y esta referencia dentro del texto agrega otra dimensión al espacio que ocupa el narrador. Dentro de tal espacio narrativo, el narrador se dirige familiarmente a un grupo designado y la categorización de vosotros implica que hay una intimidad y más bien una comunidad que percibe el narrador. Además, la narración reduce el grupo, y se dirige más específicamente a los del grupo de vosotros que tienen en común la experiencia personal de la pareja, es decir, "los que no sois solteros ni viudos." (Hernández
1914: 6) Curiosamente esta designación aunque es aparentemente exclusiva, como aparece en el texto también se puede considerar otro paso hacia universalizar la experiencia particular que está descrita en el primer párrafo. Es decir, en el primer párrafo la experiencia es entre el narrador y la lluvia, pero en el segundo, el narrador invita a un grupo familiar y pequeño a entender la experiencia inicialmente presentada desde el punto de vista individual. Es más, la invitación del narrador está reforzada por medio del uso del apóstrofe; si fuera solamente una frase, el narrador no invitaría sino asumiría y no habría oportunidad de participar. Ciertamente, hay dos apóstrofes en el segundo párrafo y porque son preguntas evitan la certeza de la expresión individual y a la vez agregan más individuos al discurso. Ahora la observación de la caída de la lluvia no es experiencia de un individuo sino de varios; no es el individuo que puede tener la experiencia con la lluvia sino también son las parejas y en los apóstrofes el narrador ofrece ejemplos de momentos plausiblemente universales entre las parejas. Como resultado, la interacción entre la pareja está en el mismo nivel de la experiencia que uno(s) tiene(n) con la lluvia. Además, los dos apóstrofes idealizan la lluvia como fuerza potente, capaz de inspirar interacciones y experiencias entre los individuos. Así, la experiencia catalizada por la lluvia juega entre lo corriente y lo nostálgico, y revela una relación binaria que va desdoblándose entre todos los elementos al leer la prosa poética. Por ejemplo, primero hay la interacción entre la lluvia y el narrador, en el presente; luego, encontramos la experiencia del narrador junto con el vosotros y la lluvia, en el presente; y luego, el narrador reconoce lo que experimentan las parejas por la lluvia, en el pasado nostálgico. Estas relaciones binarias también surgen en los apóstrofes. Al considerar el primer apóstrofe, la música de la caída de la lluvia junto con la música del cierzo inspiran una la comunicación sin palabras, en forma de una mirada entre amantes. Luego en el segundo apóstrofe, la interacción implicada entre amantes está desarrollada y traza una cadena que empieza con la caída de la lluvia: la música de la lluvia hace que reconozcan la 
música del cierzo y el ruido en las puertas, luego abre paso a la mirada compartida entre amantes, y finalmente la mujer busca conexión física con el hombre y se acomoda en los hombros del esposo, en una noche de lluvia.

Desde el segundo párrafo y luego a continuación en el tercero, se nota que el narrador no se siente solo en su impresión y por eso reconoce a los otros que comparten experiencias parecidas. Sigue dirigiéndose al grupo de vosotros y advierte la importancia y la potencia de la lluvia en relación con lo sentimental amoroso. La experiencia universal de la caída de la lluvia se amplifica con la introducción de una nueva e incipiente ideología que asimismo fue catalizada por la caída de la lluvia:

\begin{abstract}
Observad que durante la lluvia es cuando mejor se recuerda: los goces, las congojas, los ensueños y las desesperaciones pasadas, sacuden nuestra memoria con inquietud agradable entonces, como si todas las películas de nuestra vida pretérita se reflejasen, de súbito y en bien ordenada sucesión, en la tela de recuerdo. Surge-como en la magia de un encanto-de entre las nieblas de la memoria, la mujer sincera y angélica que amamos en nuestras mocedades. Con una sonrisa mitad pesar, mitad contenta, parece reprochar nuestra ingratitud, nuestro olvido...Pasa. (Hernández 1914: 6).
\end{abstract}

A primera vista, la cita del tercer párrafo parece como el recuerdo universal de los hombres que se han enamorado en la juventud. La voz narrativa parece destacar una memoria que tiene el narrador e intenta ubicarla en una tradición cotidiana, común y universal. Pero la cita también revela los temas de dualidad, de lo binario, de lo contiguo y de lo contingente que surgen en la obra de Hernández, con el catalizador común de la naturaleza. Es decir, la lluvia funciona como el puente entre la realidad y la memoria y la memoria es el puente entre el hombre y la mujer del pasado. Porque la narración se dirige a un colectivo de la segunda persona y la observación que comparte con sus interlocutores está en un presente poético establece lo que Ángel Rama identifica como una: "Ubicación histórica que permite al objeto absorber la vida cotidiana del poeta.” (Rama 1970: 116) Sugiero que el citado objeto no es singular, sino es un colectivo que incluye tanto al narrador como el vosotros en una fraternidad. Asimismo, colectivamente el narrador y el vosotros forman el objeto de la narración. En breve, los dos primeros párrafos presentan la sugerencia que hay una fraternidad que se establece por medio de experimentar la caída de la lluvia. Si consideramos el hecho de que los hermanos nacen de la misma agua materna, la lluvia, porque es una forma de agua, parece ser tan potente que puede (re)construir un tipo de fraternidad y así se establece una vía de perseguir una identidad. Por otro lado, la caída de la lluvia inspira la sentimentalidad amorosa en el narrador y pone de relieve la relación entre el hombre y la mujer idolatrada y recordada (Hernández 1914: 6). Sugiere que la fraternidad establecida por medio de la lluvia no es la única manera de (re)construirla, sino también la experiencia amorosa tiene la misma potencia. En total, se nota que la humanidad está situada como objeto de la acción de la lluvia: la lluvia inspira la memoria individual y colectiva de la pasión amorosa; la lluvia inspira el recuerdo y es la lluvia que establece una vía de identidad por medio de la experiencia universal.

En el tercer párrafo el narrador ofrece otra manifestación del poder de la lluvia porque la lluvia cataliza una reacción sentimental e íntima dentro del narrador pero el narrador todavía se dirige al vosotros. Entonces, la idea es que la experiencia de la lluvia se va experimentando simultáneamente entre varios individuos. El tercer párrafo destaca que la experiencia con la lluvia es de varios pasos: es personal, del individuo; luego es la experiencia inter-personal, entre amantes; pero también es una experiencia comunal, que aunque es particular y personal, es también universal. Y para reforzar la idea de lo comunal y universal, el narrador alista las emociones plausibles “(...) los goces, las congojas, los ensueños y las desesperaciones pasadas" (Hernández 1914: 6). La memoria del individuo es otra frontera del toco de la lluvia junto con el barrio, la casa y el espacio del narrador y sus compañeros de experiencia. En vez de la narración en primera persona singular, 
surge un inter-juego entre el vosotros y el nosotros.

Es en este tercer párrafo que se nota que hay tres palabras que marcan un proceso nostálgico: "observad", "surge" y "pasa." (Hernández 1914: 6) Estas tres palabras hacen eco a los párrafos anteriores y más bien revelan la manera en que el proceso se desarrolla desde el punto de vista del narrador. La observación de algo externo inspira el surgimiento de memorias, un proceso interno, y luego de manera repentina, pasa al olvido. El mandato seguido por los verbos en el presente indicativo trabajan para representar una situación de causa y efecto: el mandato es el catalizador que contribuye al proceso del surgimiento de la memoria, y luego el pasar de la memoria al olvido. El catalizador de la observación, porque aparece en forma de mandato, es más bien animador, y los otros verbos de "surge" y "pasa" que aparecen el presente de indicativo implican la inevitabilidad de la repetición, igual como los procesos naturales.

El cuarto párrafo, apoya al tercero, y más importantemente vincula el proceso sentimental con el proceso natural de la lluvia con la ayuda de la descripción de otro proceso natural, la del enflorecer de una rosa al salir el sol. Y más específicamente, el cuarto párrafo refuerza la idea de lo repetitivo natural, porque "...al rumor de la lluvia recordamos...” (Hernández 1914: 6) o sea el texto mismo implica el ciclo y reconoce la lluvia como catalizador.

Los párrafos anteriores abren paso al establecimiento de la ideología del narrador que se basa claramente en lo climático:

Todo convida a pesar que en los días de invierno, el ruido que produce el agua del cielo al caer sobre las cosas bajas del mundo; la inquietud que adormece el espíritu y la humedad de la temperatura excitan la sensibilidad, debilitan la intensidad de las pasiones de los humanos, y les predisponen a la recordación del pasado. (Hernández 1914: 6).

Esta cita del quinto párrafo, reconoce la lluvia como catalizador y pone a los humanos en el mismo nivel de todas las cosas del mundo.
Es más, se notan los inicios de la transición del hombre (de la humanidad) en objeto que recibe el efecto de la caída de la lluvia. Pero a continuación el texto va más allá y presenta una justificación, o más bien una explicación y manipula cómo la caída de la lluvia inspira la memoria:

Luego, la fantasía reproduce contristadoras escenas en las cuales sólo actúan seres más o menos desconocidos para nosotros [...] los niños huérfanos [...] en esos pobrezuelos, Con el viejo [...] esos desgraciados [...] esos miserables [...] la inexperta doncella, iinfeliz paloma! (Hernández 1914: 7)

Los tres párrafos finales que terminan la obra enfocan completamente en el resultado de la acción de la lluvia, inclusive una transformación en la percepción de la lluvia. No es solamente la lluvia sino el narrador que lo etiqueta "el agua del cielo" y reconoce otra manera de considerar la interacción. Con la frase “...el ruido que produce el agua del cielo al caer con las cosas bajas del mundo..." (Hernández 1914: 7) la implicación es que hay una jerarquía entre la lluvia y el mundo. La lluvia es del cielo y el mundo y todo lo que contiene está debajo. Agrega una consideración vertical al texto y desde este punto, como si fuera fuerza divina, parece que la humedad producida excita, debilita y facilita el acto interno de recordar.

El sexto párrafo explora unos recuerdos específicos peros plausiblemente universales. Como el texto menciona la fantasía junto con la memoria, creo que la memoria pertenece al narrador, y la fantasía es la invitación para que el lector participe en la memoria personal del narrador. Es también en el sexto párrafo donde el espacio del narrador añade a otros individuos; tales individuos no pertenecen ni al vosotros ni al nosotros, sino el texto los etiquetan “... seres más o menos desconocidos..." (Hernández 1914: 7) y el resto de la prosa poética se dedica a conjeturar los tipos de seres desconocidos y sus posibles situaciones tristes.

El texto termina con una idea de totalidad en el mundo: 
En los afligidos enfermos, pálidos de anemia y febriles de impaciencia; en esos desventurados que olvidados de los dioses del bien, se retuercen ora mudos, ya quejosos, creyendo que la lluvia que vendrá con el día venturo, les encontrará ya exánimes y ocultos en el negro seno de la gran madre Tierra. (Hernández 1914: 7)

El párrafo final se dirige a un nosotros entonces el apóstrofe se convierte en una autoreflexión. El párrafo final conjetura sobre la identidad y las situaciones particulares de los desconocidos en comparación de los conocidos del mencionado nosotros. En unas frases, el narrador reconoce que la lluvia cataliza la transmutación de la memoria del hombre. La memoria del narrador, se transforma en la memoria de la colectiva y porque es una memoria de un grupo puede abarcar "...seres más o menos desconocidos...” (Hernández 1914: 7) Los seres desconocidos ocupan una lista igual como las otras cosas del mundo y todos padecen bajo el mismo efecto de la lluvia. El texto termina en la transmutación más profunda que se puede concebir que es la incorporación en la tierra, o sea la muerte. Se nota que el camino de la transmutación no es un continuo sino se revela como un ciclo. Empieza con la realización existencial de la lluvia, luego el reconocimiento de la caída de la lluvia, luego el relato presencial del narrador y finalmente el reconocimiento de que por una experiencia en común, puede también haber una memoria en común. A base de esa exploración de la identidad por medio de lo contiguo en la experiencia de la lluvia nace otra dimensión de la identidad.

En aquel entonces del ambiente modernista de Panamá, el nacionalismo y la identidad panameña eran asuntos principales. Porque el modernismo coincide con los primeros años de la República de Panamá, y porque el Istmo llevaba años de intervención extranjera, el establecer una identidad era imprescindible. Tomando la importancia de la identidad panameña en cuenta el próximo paso es establecer un ancla para acercarse críticamente a la obra de Hernández. En la crítica de Ángel Rama encontré un acercamiento compatible. En su artículo "Indagación le la ideología en la poesía (Los Dípticos Seriados de Versos Sencillos)" y en su libro Rubén Dario y el modernismo, pude formar una aproximación, aunque Rama enfoca en la obra de Rubén Darío. Ahora bien, Rama por medio de su crítica de Darío y del modernismo, destaca dos conceptos literarios que contribuyen al modernismo. En su libro, Rubén Dario y el modernismo, Rama discurre sobre un concepto que es la subjetivación, y ofrece la siguiente definición:

Su rasgo clave es la 'subjetivación' ascendida a
valor único, [...] Eso significa imponer [...] una
fractura entre el mundo y el hombre, que [...]
remite drásticamente la existencia del universo al
padecimiento y a la gloria de una consciencia que
se aísla y se le opone. (Rama 1970: 12-13)

Luego, en su artículo " Indagación le la ideología en la poesía (Los Dípticos Seriados de Versos Sencillos)" Rama discurre sobre el concepto de la transmutación en relación con los procesos naturales. Rama reconoce cómo la transmutación se manifiesta como otro aspecto particular del modernismo:

La pérdida de la consciencia individual, del "yo"
transmuta austeramente los elementos naturales
en juego, situándolos por de fuera de los sistemas
valorativos culturales, haciendo de ellos simples
objetos de la realidad [...] Esta afirmación acarrea
la evicción del "yo", [...] el hombre pasa a ser,
también objeto natural. (Rama 2009: 368)

En mi análisis he puesto los dos conceptos en un orden, para marcar pasos de un ciclo o más bien de un proceso. Los dos conceptos combinados de textos distintos proveen la clave de entender cómo aproximarnos a esta obra de Hernández. Como guía, diseñé el diagrama abajo que traza cómo estos conceptos se desarrollan en la poesía en prosa de "Lluvia;" el diagrama también funciona como guía para considerar el tipo de modernismo que encontramos en la obra de Hernández y quizás se aplicaría al considerar las obras modernistas de sus contemporáneos panameños también. 


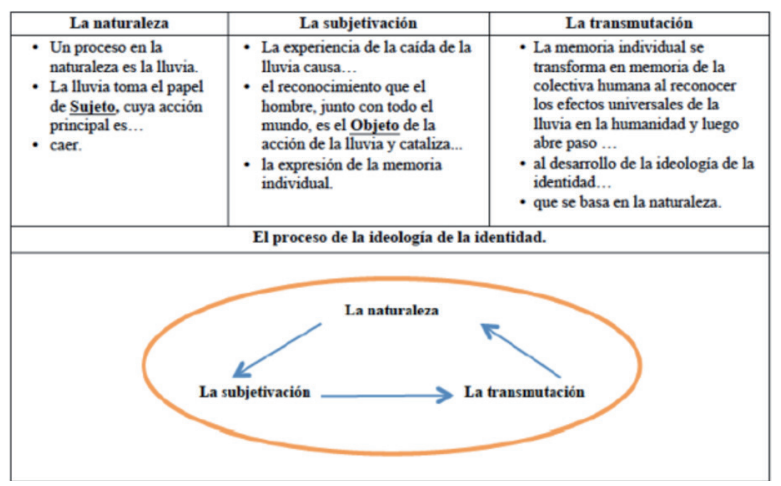

FIGURA 1.1

La búsqueda de la identidad en la prosa poética de "Lluvia", por Gaspar Octavio Hernández, empleando una combinación de la crítica de Ángel Rama.

Al estudiar el diagrama, se nota que los dos conceptos, la subjetivación y la transmutación ocurren en la obra de "Lluvia" y el rasgo único es la predominancia de la naturaleza en el discurso. Al reconocer el efecto de la lluvia y la subjetivación en la experiencia, la ideología de la identidad empieza a revelarse. Se da cuenta que la lluvia no es solamente una ocurrencia temporal sino es un catalizador para poder ubicarse en el dolor de la memoria o quizás para ubicarse en la memoria del dolor. Como resultado la obra de Hernández mantiene un discurso que se enfoca en la búsqueda de la identidad y asume que el hombre es sólo un objeto en el conjunto de la naturaleza. Las citas de Rama en cuanto a la subjetivación (Rama 1970: 12-13) y la transmutación (Rama 2009: 368) junto con el reconocimiento de la importancia de la naturaleza en el modernismo panameño en este análisis, trabajan para sintetizar los tres motivos del texto de "Lluvia": primero, presentar la naturaleza en forma de lluvia; segundo, presentar la subjetivación del narrador y el impresionismo de cómo experimenta la lluvia; y finalmente, la transmutación catalizada por la caída de la lluvia. El resultado es una ideología que establece a su colmo, al hombre como objeto dentro de la naturaleza. La implicación al final de texto es que la naturaleza es integral en la ideología de la identidad panameña.
En cuanto a la identidad panameña, no es evidente si el texto conteste la cuestión, pero sí que añade una manera de encontrar un punto de partida. El medioambiente y los procesos en sí son aspectos que pertenecen a todo el mundo pero al considerar la situación de Panamá, esos aspectos son principales en la búsqueda de su identidad. El país y todo lo relacionado con su situación mundial hacen al Istmo de Panamá un país único. En su obra de poesía en prosa Hernández logró escudriñar los aspectos particulares a Panamá en cuanto a procesos temporales y por medio de su descripción multifacética provee un inicio donde ubicarnos como humanos dentro del mundo. Es evidente que la naturaleza y el medioambiente panameño sirvieron como fundamentos de (re) formar una identidad en el contexto personal de Hernández tanto como el contexto histórico de Panamá. También es evidente que aunque no es claramente probado si llegó a una impresión adecuada de la identidad personal y/o panameña, es evidente Hernández mantiene como prioridad la búsqueda de la identidad.

En esta obra la imagen de la lluvia y la interacción que tiene con los sujetos de la tierra y del hombre funcionan como ancla de expresión dentro de la prosa poética. Por medio del diálogo interior y un discurso existencialista se revela que la voz narrativa se siente últimamente conectada 
con la naturaleza y más específicamente se siente vinculada con los procesos de la naturaleza. Para Hernández, la idea de la identidad podría ser múltiple: primero sería un reconocimiento de la naturaleza, (en este caso la lluvia) que abría paso a la novedad de reconsiderar el papel del hombre en el mundo (en vez de sujeto, es objeto), que resultaría en una ideología que se basa en el la naturaleza. Sin embargo, se revela que la clave del ciclo entiende que la búsqueda de la identidad tanto empieza como termina en la naturaleza. En el texto se nota que a partir de reconocer lo real de la experiencia de la lluvia, no hay una sensación escapista, sino hay una cierta sensación de que el narrador por el impacto del clima y el acontecimiento temporal, se sienta motivado a relatar la experiencia ambiental con un discurso universalizado por medio de referencias naturales. En breve, este texto evidencia que Hernández considera la naturaleza como la explicación más apropiada en cuanto a cualquier cuestión sobre la identidad.

\section{Notas}

1. En su trabajo extensivo, Rosendo González Batista traza la historia del periodismo en Panamá con una concentración en las publicaciones académicas y científicas que se encuentran en la Biblioteca Nacional Ernesto J. Castillero en la ciudad de Panamá. Aunque el trabajo es más bien una de índole científica, me sirvió mucho al compilar una línea de hechos histórico que tendría impacto en cuanto a modernismo y los movimientos anteriores. El estudio incluye información desde el año 1821 hasta el 2003.

2. Durante esta época Panamá había inestabilidad en cuanto a lo que Araúz y Pizzurno señalan "El revisionismo y la lucha por la soberanía y la integridad territorial" (Araúz 1996: 84-88). Básicamente, durante la construcción y hasta la apertura del Canal estaban tratando de re-negociar las estipulaciones del tratado Hay-Bunau-Varilla, que resultaron en la falta de propiedad panameña de la zona alrededor del Canal. Específicamente, el artículo II del tratado “...concedió a perpetuidad a Estados Unidos, el uso, ocupación y control de cualesquiera otras tierras y aguas fuera de la Zona del Canal que pudieran se necesarias y convenientes para construcción, saneamiento y protección de la vía intermarina...Así cuando se llevaron a cabo las obras de construcción de la ruta, el gobierno estadounidense exigió la entrega de tierras y aguas fuera de la Zona del Canal, y procedió a expropiar las misma, aunque el Gobierno de Panamá, en ocasiones, mostró disconformidad." (Araúz 1996: 88) Es muy posible que la sociedad panameña se sintiera cierto resentimiento contra los intereses estadounidenses, al ver la explotación gratuita de la tierra y agua panameña.

3. La generación de escritores llevan ese título en el La literatura panameña por Rodrigo Miró.

\section{Bibliografía}

Araúz, Celestino A. y Gelós P. Pizzurno. 1996. Estudios Sobre El Panamá Republicano: 1903-1989. Panamá: Manfer.

Geenzier, Enrique y Santiago L. Benuzzi, eds. 1914. Esto y Aquello, 1, 15 agosto 1914.

González Batista, Rosendo. 2009. Concentración Dispersión de la Información en Revistas Académicas y Científicas en la Bibligrafía Nacional de Panamá. Universidad de Panamá.

Hernández, Gaspar O. Lluvia. Esto y Aquello 15 agosto 1914: 6-7. Print.

1916. Iconografía. Panamá: Esto y Aquello.

Miró, Rodrigo. 1975. Itinerario de la poesía en Panamá: 1502-1974. Panamá: Editorial Universitaria.

1996. La Literatura Panameña: Origen y Proceso. Panamá: Editorial Serviprensa.

Peña, Concha. Gaspar Octavio Hernández: Poeta Del Pueblo. Panamá: s.n., 1953. 
Rama, A. 2009. Indagación de la ideología en la poesía (Los Dípticos Seriados de Versos Sencillos). Revista Iberoamericana, 46, 112.
1970. Rubén Dario y el Modernismo (Circunstancia Socioeconómica De Un Arte Americano). Caracas: Ediciones de la Biblioteca de la Universidad Central de Venezuela.

\section{(ब) $\Theta \Theta$}


